\title{
An Improved Unscented Kalman Filter for Discrete Nonlinear Systems with Random Parameters
}

\author{
Yue Wang, ${ }^{1}$ Zhijian Qiu, ${ }^{1}$ and Xiaomei Qu ${ }^{2}$ \\ ${ }^{1}$ School of Economic Mathematics, Southwestern University of Finance and Economics, Chengdu, Sichuan 611130, China \\ ${ }^{2}$ College of Computer Science and Technology, Southwest University for Nationalities, Chengdu, Sichuan 610041, China \\ Correspondence should be addressed to Yue Wang; wangyue_001@163.com
}

Received 29 December 2016; Accepted 6 February 2017; Published 26 February 2017

Academic Editor: Delfim F. M. Torres

Copyright (c) 2017 Yue Wang et al. This is an open access article distributed under the Creative Commons Attribution License, which permits unrestricted use, distribution, and reproduction in any medium, provided the original work is properly cited.

\begin{abstract}
This paper investigates the nonlinear unscented Kalman filtering (UKF) problem for discrete nonlinear dynamic systems with random parameters. We develop an improved unscented transformation by incorporating the random parameters into the state vector to enlarge the number of sigma points. The theoretical analysis reveals that the approximated mean and covariance via the improved unscented transformation match the true values correctly up to the third order of Taylor series expansion. Based on the improved unscented transformation, an improved UKF method is proposed to expand the application of the UKF for nonlinear systems with random parameters. An application to the mobile source localization with time difference of arrival (TDOA) measurements and sensor position uncertainties is provided where the simulation results illustrate that the improved UKF method leads to a superior performance in comparison with the normal UKF method.
\end{abstract}

\section{Introduction}

Since Kalman proposed his famous recursive method to solve discrete dynamic filtering problems [1], the Kalman filter has been widely used in many areas, ranging from engineering to economics $[2,3]$. However, the linearity of the dynamic system, as one of the basic requirements of the Kalman filter, is hard to satisfy in actual implementation. On the other hand, discrete dynamic systems with random parameters arise in many applications such as missile track estimation, satellite navigation, maneuvering target tracking, and economic forecast [4-8].

For the linear discrete dynamic system where the state transition and measurement matrices are random parameters, De Koning [4] provides a linear minimum variance recursive estimator without rigorous derivation. By converting the system to a linear dynamic system with deterministic parameter matrices and state-dependent noises, Luo et al. [6] propose the optimal linear filtering with the form of a modified Kalman filtering.

Unlike the filtering problem in linear dynamic systems with random parameters, which has been extensively studied, the research on the filtering for nonlinear dynamic systems with random parameters has seldom been reported in the literature. The main challenge lies in how to transform a probability density function (pdf) through a general nonlinear function which contains random parameters.

In order to make the Kalman filter applicable to nonlinear dynamic systems, the extended Kalman filter (EKF) [9], based on the first-order approximation in the Taylor series expansion of a nonlinear function, is proposed. Although the EKF method maintains the computationally efficient update form of the Kalman filter, its estimation accuracy may be unsatisfactory due to neglecting the higher-order terms of nonlinear system function [10]. Also, the EKF requires the calculation of the Jacobian matrix, which is difficult to implement in practical applications [11].

Since the seminal work of Julier et al. in 1995 [12], the unscented Kalman filter (UKF), which is an extension of the Kalman filter, reducing the linearization errors of the EKF, has been an object of great interest in nonlinear filtering [13-17]. The UKF is a derivative-free filter which combines the concept of unscented transformation with the linear update structure of the Kalman filtering [12]. In addition, the 
UKF method is based on statistical approximations of system equations without requiring the calculation of the Jacobian matrix [13].

The fundamental of UKF is the unscented transformation which uses a set of sigma points to propagate the mean and covariance matrix [13]. The sigma points are deterministically calculated from the mean and square-root decomposition of the covariance matrix of the a priori random variable. By applying the nonlinear system function to each sigma point to obtain transformed vectors, the ensemble mean and covariance of the transformed vectors give an estimate of the true mean and covariance. In theory, if the nonlinear system function is completely specified, the unscented transformation can capture the posterior mean and covariance of a random variable with symmetric pdf accurately up to the third order of Taylor series expansion [18], leading to a superior performance in comparison with the EKF method.

However, when the nonlinear system function contains random parameters, the step of transforming the sigma points to transformed vectors is unrealizable. One method to deal with this problem is to neglect the randomness of these parameters by using the nominal parameters in the transforming procedure. In intuition, this will lead to unexpected error in the corresponding unscented transformation, especially when the true parameters are far from the nominal parameters.

The key motivation of this paper is to overcome the difficulty of unscented transformation with random parameters and expand the application of the UKF for the nonlinear state estimation. Hence, we focus on the design of an improved unscented transformation by regarding the random parameters as part of the state to augment the state vector. In the corresponding improved unscented transformation, the number of sigma points is enlarged due to the increased dimension of the state vector. In addition, each improved sigma point contains samples of both the state and the random parameters, so there are no unknown parameters involved in the step of transforming the sigma points.

Our main contributions in this paper include the following: (i) An improved unscented transformation and the corresponding improved UKF method are proposed to deal with the nonlinear dynamic system with random parameters; (ii) the performance analysis is provided which shows that the approximated mean and covariance via the improved unscented transformation match the true values correctly up to the third order of Taylor series expansion when the nonlinear system function contains random parameters.

An application of the proposed improved UKF method to the mobile source localization problem is provided, where we consider source localization by a network of passive sensors using noisy time difference of arrival (TDOA) measurements. Extensive studies for the static source localization by TDOA measurements can be found in $[19,20]$. However, in practice, the emitter source may not be static, which could be mounted on-board of dynamic mobile platforms [21]. In addition, accurate sensor location information may not be available [20]. For instance, in modern localization applications, sensors could be deployed randomly in a field and their nominal locations may not be accurate. The mobile source localization problem with sensor location uncertainties can be described as a discrete nonlinear dynamic system with random parameters.

The Monte Carlo simulations are carried out to compare the localization performance of the improved UKF method and the normal UKF method. The results indicate that the root of mean squared error (RMSE) of mobile localization is evidently decreased by the improved UKF method, especially when the sensor position uncertainty level is high, which corroborates that the proposed improved UKF method can improve the estimation accuracy of the nonlinear dynamic systems with random parameters.

The remainder of the paper is organized as follows. Section 2 briefly describes the nonlinear system model and the unscented Kalman filter. Section 3 presents the improved unscented Kalman filtering method for the nonlinear dynamic system with random parameters. An application to the mobile source localization problem and the Monte Carlo simulation results are provided in Section 4 and Section 5 gives some concluding remarks.

Throughout this paper, the transpose and inverse of matrix $\mathbf{X}$ are denoted by $\mathbf{X}^{\prime}$ and $\mathbf{X}^{-1}$, respectively, the "-" and "+" superscripts denote that the estimate is a priori and a posteriori estimate, respectively, $\|\cdot\|$ means the Euclidean norm, and $E(\cdot)$ is the mathematical expectation.

\section{Nonlinear and Unscented Kalman Filter}

The unscented Kalman filter provides a suboptimal solution for the stochastic filtering problem of a nonlinear discretetime, dynamic system in the form

$$
\begin{aligned}
& \mathbf{x}_{k}=f\left(\mathbf{x}_{k-1}\right)+\mathbf{w}_{k}, \\
& \mathbf{y}_{k}=h\left(\mathbf{x}_{k}\right)+\mathbf{v}_{k},
\end{aligned}
$$

where $k$ is the discrete-time instant, $\mathbf{x}_{k} \in \mathbb{R}^{n_{x}}$ is the state vector, $\mathbf{y}_{k} \in \mathbb{R}^{n_{y}}$ is the measurement output, and $f(\cdot)$ and $h(\cdot)$ are the process and measurement functions, respectively. The vectors $\mathbf{w}_{k}$ and $\mathbf{v}_{k}$ are two zero-mean white Gaussian noise processes with covariance matrixes $\mathbf{Q}_{w}$ and $\mathbf{Q}_{v}$, respectively. The noise terms $\mathbf{w}_{k}$ and $\mathbf{v}_{k}$ are assumed to be uncorrelated.

The goal of the stochastic filtering is to estimate the state $\mathbf{x}_{k}$ as new measurements $\mathbf{y}_{k}$ are acquired. When the process and measurement functions are linear to the state vector, the celebrated Kalman filter provides the optimal solution with respect to the minimum variance (MV) criterion [22]. However, in the case of nonlinear systems, such optimal solution tends to be computationally intractable [18, 23]. Therefore, suboptimal approaches are developed such as extended Kalman filter (EKF) [9] and unscented Kalman filter (UKF) [13].

The EKF works on the principle that a linearized transformation of means and covariances is approximately equal to the true nonlinear transformation, but this approximation could be unsatisfactory in practice. In the following, we will briefly describe the unscented transformation which is the key technique of the UKF. 
2.1. Unscented Transformation. Suppose that we have a vector $\mathbf{x} \in \mathbb{R}^{n_{x}}$ with known mean $\overline{\mathbf{x}}$ and covariance $\mathbf{P}$, and its pdf is symmetric around its mean vector. For a nonlinear function $\mathbf{y}=h(\mathbf{x})$, we want to approximate the mean and covariance of $\mathbf{y}$. The first step of the unscented transformation is to find a set of deterministic vectors called sigma points whose ensemble weighted mean and covariance are equal to $\overline{\mathbf{x}}$ and $\mathbf{P}$, respectively, which can be realized as follows:

$$
\mathbf{x}^{(j)}=\overline{\mathbf{x}}+\widetilde{\mathbf{x}}^{(j)}, \quad j=1, \ldots, 2 n_{x},
$$

where

$$
\begin{gathered}
\widetilde{\mathbf{x}}^{(j)}=\left(\sqrt{n_{x} \mathbf{P}}\right)_{j}^{\prime}, \quad j=1, \ldots, n_{x}, \\
\widetilde{\mathbf{x}}^{\left(n_{x}+j\right)}=-\left(\sqrt{n_{x} \mathbf{P}}\right)_{j}^{\prime}, \quad j=1, \ldots, n_{x},
\end{gathered}
$$

$\sqrt{n_{x} \mathbf{P}}$ is the matrix square root of $n_{x} \mathbf{P}$ such that $\left(\sqrt{n_{x} \mathbf{P}}\right)^{\prime} \sqrt{n_{x} \mathbf{P}}=n_{x} \mathbf{P}$, and $\left(\sqrt{n_{x} \mathbf{P}}\right)_{j}$ is the $j$ th row of $\sqrt{n_{x} \mathbf{P}}$. The weighting coefficients are given as

$$
\mathbf{W}^{(j)}=\frac{1}{2 n_{x}}, \quad j=1, \ldots, 2 n_{x} .
$$

Then, we transform each sigma point in (2) using the nonlinear function $h(\cdot)$ and approximate the mean of $\mathbf{y}$ by taking the weighted sum of the transformed sigma points. Specifically, let $\overline{\mathbf{y}}$ denote the true mean of $\mathbf{y}$. The approximated mean of $\mathbf{y}$ is denoted as $\hat{\overline{\mathbf{y}}}$ and computed as follows:

$$
\widehat{\overline{\mathbf{y}}}=\sum_{j=1}^{2 n_{x}} \mathbf{W}^{(j)} \mathbf{y}^{(j)}, \quad \mathbf{y}^{(j)}=h\left(\mathbf{x}^{(j)}\right), j=1, \ldots, 2 n_{x} .
$$

Let $\overline{\mathbf{P}}_{\mathbf{y}}$ denote the true covariance of $\mathbf{y}$. The approximated covariance of $\mathbf{y}$ is denoted as $\widehat{\overline{\mathbf{P}}}_{\mathbf{y}}$ and computed as follows:

$$
\widehat{\overline{\mathbf{P}}}_{\mathbf{y}}=\sum_{j=1}^{2 n_{x}} \mathbf{W}^{(j)}\left(\mathbf{y}^{(j)}-\widehat{\overline{\mathbf{y}}}\right)\left(\mathbf{y}^{(j)}-\widehat{\overline{\mathbf{y}}}\right)^{\prime} .
$$

The unscented transformations (5) and (6) are more accurate than the linearization method for propagating means and covariances of nonlinear functions, which is summarized in the following proposition.

Proposition 1 (see [18]). For random vector $\mathbf{x}$ with a symmetric pdf around its mean, if the nonlinear function $\mathbf{y}=$ $h(\mathbf{x})$ is completely specified, then the approximated mean and covariance of $\mathbf{y}$ via the unscented transformations (5)-(6) match the true mean and covariance of $\mathbf{y}$ correctly up to the third order.

2.2. Unscented Kalman Filter. The unscented transformation can be generalized to give the unscented Kalman filter, which keeps the structure of the Kalman filter that includes one prediction (or a time update) step and one correction (or a measurement update) step.
For the discrete nonlinear system (1), the unscented Kalman filter is initialized as follows:

$$
\begin{aligned}
\widehat{\mathbf{x}}_{0}^{+} & =E\left(\mathbf{x}_{0}\right), \\
\mathbf{P}_{0}^{+} & =E\left[\left(\mathbf{x}_{0}-\widehat{\mathbf{x}}_{0}^{+}\right)\left(\mathbf{x}_{0}-\widehat{\mathbf{x}}_{0}^{+}\right)^{\prime}\right] .
\end{aligned}
$$

Suppose that, at time step $k, \widehat{\mathbf{x}}_{k-1}^{+}$and $\mathbf{P}_{k-1}^{+}$are given. The UKF algorithm can be summarized in Algorithm 2.

Algorithm 2 (the unscented Kalman filter).

(1) Choose the sigma points $\widehat{\mathbf{x}}_{k-1}^{(j)}$ as specified in (2) with appropriate changes, that is,

$$
\begin{gathered}
\widehat{\mathbf{x}}_{k-1}^{(j)}=\widehat{\mathbf{x}}_{k-1}^{+}+\widetilde{\mathbf{x}}_{k-1}^{(j)}, \quad j=1, \ldots, 2 n_{x} \\
\widetilde{\mathbf{x}}_{k-1}^{(j)}=\left(\sqrt{n_{x} \mathbf{P}_{k-1}^{+}}\right)_{j}^{\prime}, \quad j=1, \ldots, n_{x}, \\
\widetilde{\mathbf{x}}_{k-1}^{\left(n_{x}+j\right)}=-\left(\sqrt{n_{x} \mathbf{P}_{k-1}^{+}}\right)_{j}^{\prime}, \quad j=1, \ldots, n_{x} .
\end{gathered}
$$

(2) Use the completely specified motion function $f(\cdot)$ to transform the sigma points into $\widehat{\mathbf{x}}_{k}^{(j)}$ vectors:

$$
\widehat{\mathbf{x}}_{k}^{(j)}=f\left(\widehat{\mathbf{x}}_{k-1}^{(j)}\right) \text {. }
$$

(3) Combine the $\widehat{\mathbf{x}}_{k}^{(j)}$ vectors to obtain the prediction estimate at time $k$

$$
\widehat{\mathbf{x}}_{k}^{-}=\frac{1}{2 n_{x}} \sum_{j=1}^{2 n_{x}} \widehat{\mathbf{x}}_{k}^{(j)}
$$

and the corresponding error covariance

$$
\mathbf{P}_{k}^{-}=\frac{1}{2 n_{x}} \sum_{j=1}^{2 n_{x}}\left(\widehat{\mathbf{x}}_{k}^{(j)}-\widehat{\mathbf{x}}_{k}^{-}\right)\left(\widehat{\mathbf{x}}_{k}^{(j)}-\widehat{\mathbf{x}}_{k}^{-}\right)^{\prime}+\mathbf{Q}_{w} .
$$

(4) Use the completely specified measurement function $h(\cdot)$ to transform the sigma points into $\widehat{\mathbf{y}}_{k}^{(j)}$ vectors:

$$
\widehat{\mathbf{y}}_{k}^{(j)}=h\left(\widehat{\mathbf{x}}_{k-1}^{(j)}\right)
$$

(5) Combine the $\widehat{\mathbf{y}}_{k}^{(j)}$ vectors to obtain the predicted measurement at time $k$

$$
\widehat{\mathbf{y}}_{k}=\frac{1}{2 n_{x}} \sum_{j=1}^{2 n_{x}} \widehat{\mathbf{y}}_{k}^{(j)}
$$

and the corresponding covariance

$$
\mathbf{P}_{k}^{y}=\frac{1}{2 n_{x}} \sum_{j=1}^{2 n_{x}}\left(\widehat{\mathbf{y}}_{k}^{(j)}-\widehat{\mathbf{y}}_{k}\right)\left(\widehat{\mathbf{y}}_{k}^{(j)}-\widehat{\mathbf{y}}_{k}\right)^{\prime}+\mathbf{Q}_{v} .
$$


(6) Estimate the cross covariance between $\widehat{\mathbf{x}}_{k}^{-}$and $\widehat{\mathbf{y}}_{k}$ :

$$
\mathbf{P}_{k}^{x y}=\frac{1}{2 n_{x}} \sum_{j=1}^{2 n_{x}}\left(\widehat{\mathbf{x}}_{k}^{(j)}-\widehat{\mathbf{x}}_{k}^{-}\right)\left(\widehat{\mathbf{y}}_{k}^{(j)}-\widehat{\mathbf{y}}_{k}\right)^{\prime} .
$$

(7) The measurement update of the state estimate can be performed following the normal Kalman filter:

$$
\begin{aligned}
\mathbf{K}_{k} & =\mathbf{P}_{k}^{x y}\left(\mathbf{P}_{k}^{y}\right)^{-1}, \\
\widehat{\mathbf{x}}_{k}^{+} & =\widehat{\mathbf{x}}_{k}^{-}+\mathbf{K}_{k}\left(\mathbf{y}_{k}-\widehat{\mathbf{y}}_{k}\right), \\
\mathbf{P}_{k}^{+} & =\mathbf{P}_{k}^{-}-\mathbf{K}_{k} \mathbf{P}_{k}^{y} \mathbf{K}_{k}^{\prime} .
\end{aligned}
$$

The algorithm above assumes that the motion and measurement functions are completely known. In practical applications, the motion and measurement functions may contain parameters that are not specified and may be random with known distributions. In such cases, the previous UKF algorithm can not be directly applied.

\section{Improved Unscented Kalman Filter}

In this section, we propose an improved UKF method to deal with the filtering problem in nonlinear dynamic systems with random parameters. In the following, we will firstly introduce the improved unscented transformation which propagates the mean and covariance of a random vector through a nonlinear function with random parameters.

3.1. Improved Unscented Transformation. Suppose that we have a vector $\mathbf{x} \in \mathbb{R}^{n_{x}}$ with known mean $\overline{\mathbf{x}}$ and covariance $\mathbf{P}$, and its pdf is symmetric around its mean vector. For a nonlinear function $\mathbf{y}=h(\mathbf{x}, \theta)$, the random parameter $\theta \epsilon$ $\mathbb{R}^{n_{\theta}}$ has known mean $\bar{\theta}$ and covariance $\mathbf{P}^{\theta}$ and also has a symmetric pdf. In addition, the parameter $\theta$ is uncorrelated with $\mathbf{x}$.

In the normal unscented transformation, a set of sigma points $\mathbf{x}^{(j)}$ (2) is generated, and each sigma point is transformed by the known nonlinear function $h(\cdot)$ to approximate the mean and covariance of $\mathbf{y}$. However, when the function $h(\cdot)$ is nonlinear with respect to random parameter $\theta$, the step of transforming the sigma points is unrealizable.

In order to deal with this problem, we augment the random parameter $\theta$ onto the state vector as follows:

$$
\check{\mathbf{x}}=\left[\mathbf{x}^{\prime}, \theta^{\prime}\right]^{\prime} \in \mathbb{R}^{n_{x}+n_{\theta}} .
$$

The mean and covariance of the augment vector $\breve{\mathbf{x}}$ are

$$
\begin{gathered}
E(\check{\mathbf{x}})=\overline{\check{\mathbf{x}}}=\left[\overline{\mathbf{x}}^{\prime}, \bar{\theta}^{\prime}\right]^{\prime}, \\
\operatorname{Cov}(\check{\mathbf{x}})=\check{\mathbf{P}}=\left(\begin{array}{cc}
\mathbf{P} & \mathbf{0} \\
\mathbf{0} & \mathbf{P}^{\theta}
\end{array}\right) .
\end{gathered}
$$

Based on the augment model, the number of sigma points is enlarged to $2\left(n_{x}+n_{\theta}\right)$. The new sigma points are generated as follows:

$$
\check{\mathbf{x}}^{(j)}=\overline{\check{\mathbf{x}}}+\widetilde{\mathbf{x}}_{k}^{(j)}, \quad j=1, \ldots, 2\left(n_{x}+n_{\theta}\right),
$$

where

$$
\begin{array}{r}
\widetilde{\tilde{\mathbf{x}}}^{(j)}=\left(\sqrt{2\left(n_{x}+n_{\theta}\right) \check{\mathbf{P}}}\right)_{j}^{\prime}, j=1, \ldots, n_{x}+n_{\theta}, \\
\widetilde{\check{\mathbf{x}}}^{\left(n_{x}+n_{\theta}+j\right)}=-\left(\sqrt{2\left(n_{x}+n_{\theta}\right) \check{\mathbf{P}}}\right)_{j}^{\prime}, \\
\quad j=1, \ldots, n_{x}+n_{\theta} .
\end{array}
$$

The corresponding weighting coefficients are given as

$$
\check{\mathbf{W}}^{(j)}=\frac{1}{2\left(n_{x}+n_{\theta}\right)}, \quad j=1, \ldots, 2\left(n_{x}+n_{\theta}\right) .
$$

Because we regard both the vector $\mathbf{x}$ and the random parameter $\theta$ as the unknown parameters in the augment model, each sigma point can be divided into two parts, that is,

$$
\check{\mathbf{x}}^{(j)}=\left[\mathbf{x}^{(j)}, \theta^{(j)}\right],
$$

where $\mathbf{x}^{(j)}=\check{\mathbf{x}}^{(j)}\left(1: n_{x}\right)$ represents the sigma point of the vector $\mathbf{x}$ and $\theta^{(j)}=\check{\mathbf{x}}^{(j)}\left(n_{x}+1: n_{x}+n_{\theta}\right)$ represents the sigma point of the parameter $\theta$. As a result, the transformed sigma points can be computed as follows:

$$
\mathbf{y}^{(j)}=h\left(\mathbf{x}^{(j)}, \theta^{(j)}\right), \quad j=1, \ldots, 2\left(n_{x}+n_{\theta}\right) .
$$

Let $\overline{\mathbf{y}}$ denote the true mean of $\mathbf{y}$. The approximated mean of the improved unscented transformation is denoted as $\widehat{\overline{\mathbf{y}}}$ and computed as follows:

$$
\widehat{\overline{\mathbf{y}}}=\sum_{j=1}^{2\left(n_{x}+n_{\theta}\right)} \check{\mathbf{W}}^{(j)} \mathbf{y}^{(j)} .
$$

Let $\overline{\mathbf{P}}_{\mathbf{y}}$ denote the true covariance of $\mathbf{y}$. The approximated covariance of the improved unscented transformation is denoted as $\widehat{\overline{\mathbf{P}}}_{\mathbf{y}}$ and computed as follows:

$$
\widehat{\overline{\mathbf{P}}}_{\mathbf{y}}=\sum_{j=1}^{2\left(n_{x}+n_{\theta}\right)} \check{\mathbf{W}}^{(j)}\left(\mathbf{y}^{(j)}-\widehat{\overline{\mathbf{y}}}\right)\left(\mathbf{y}^{(j)}-\widehat{\overline{\mathbf{y}}}\right)^{\prime} .
$$

The improved unscented transformations (24) and (25) incorporate the information of the random parameter $\theta$, so they are intuitively more accurate than the normal unscented transformations (5) and (6) which ignore the randomness of $\theta$. The theoretical analysis of the improved unscented transformations is given in the following theorems.

Theorem 3. For random vector $\mathbf{x}$ with a symmetric $p d f$ around its mean, if the nonlinear function $\mathbf{y}=h(\mathbf{x}, \theta)$ contains unknown random parameter $\theta$, the approximated mean of $\mathbf{y}$ via the improved unscented transformation (24) matches the true mean of $\mathbf{y}$ correctly up to the third order.

Proof. We firstly expand $\mathbf{y}=h(\mathbf{x}, \theta)$ in a Taylor series around $\overline{\check{\mathbf{x}}}=\left[\overline{\mathbf{x}}^{\prime}, \bar{\theta}^{\prime}\right]^{\prime}$ as follows:

$$
\begin{aligned}
\mathbf{y} & =h(\mathbf{x}, \theta) \\
& =h(\overline{\mathbf{x}}, \bar{\theta})+D_{\widetilde{\mathbf{x}}} h+\frac{1}{2 !} D_{\widetilde{\mathbf{x}}}^{2} h+\frac{1}{3 !} D_{\widetilde{\mathbf{x}}}^{3} h+\cdots,
\end{aligned}
$$


where $\widetilde{\mathbf{x}}=\check{\mathbf{x}}-\overline{\check{\mathbf{x}}}$ and the operation $D_{\widetilde{\mathbf{x}}}^{k} h$ is defined as

$$
D_{\widetilde{\mathbf{x}}}^{k} h=\left(\left.\sum_{j=1}^{n_{x}+n_{\theta}} \widetilde{\mathbf{x}}_{j} \frac{\partial h}{\partial \check{\mathbf{x}}_{j}}\right|_{\check{\mathbf{x}}=\overline{\mathbf{x}}}\right)^{k} .
$$

The mean of $\mathbf{y}$ can therefore be expanded as

$$
\begin{aligned}
\overline{\mathbf{y}} & =E\left(h(\overline{\mathbf{x}}, \bar{\theta})+D_{\widetilde{\mathbf{x}}} h+\frac{1}{2 !} D_{\widetilde{\mathbf{x}}}^{2} h+\frac{1}{3 !} D_{\widetilde{\mathbf{x}}}^{3} h+\cdots\right) \\
& =h(\overline{\mathbf{x}}, \bar{\theta})+E\left(D_{\widetilde{\mathbf{x}}} h+\frac{1}{2 !} D_{\widetilde{\mathbf{x}}}^{2} h+\frac{1}{3 !} D_{\widetilde{\mathbf{x}}}^{3} h+\cdots\right) .
\end{aligned}
$$

We can see that

$$
\begin{aligned}
E\left(D_{\tilde{\mathbf{x}}} h\right) & =E\left(\left.\sum_{j=1}^{n_{x}+n_{\theta}} \widetilde{\widetilde{\mathbf{x}}}_{j} \frac{\partial h}{\partial \check{\mathbf{x}}_{j}}\right|_{\check{\mathbf{x}}=\overline{\mathbf{x}}}\right) \\
& =\left.\sum_{j=1}^{n_{x}+n_{\theta}} E\left(\widetilde{\mathbf{x}}_{j}\right) \frac{\partial h}{\partial \check{\mathbf{x}}_{j}}\right|_{\check{\mathbf{x}}=\overline{\mathbf{x}}}=0
\end{aligned}
$$

due to $E(\widetilde{\mathbf{x}})=\mathbf{0}$. Because both $\mathbf{x}$ and $\theta$ have a symmetric pdf around the mean vector, we can verify that

$$
E\left(D_{\widetilde{\mathbf{x}}}^{3} h\right)=E\left[\left(\left.\sum_{j=1}^{n_{x}+n_{\theta}} \widetilde{\mathbf{x}}_{j} \frac{\partial h}{\partial \check{\mathbf{x}}_{j}}\right|_{\check{\mathbf{x}}=\overline{\mathbf{x}}}\right)^{3}\right]=0
$$

Similarly, all of the odd terms in (28) will be equal to zero, which leads to the simplification

$$
\overline{\mathbf{y}}=h(\overline{\mathbf{x}}, \bar{\theta})+E\left(\frac{1}{2 !} D_{\widetilde{\mathbf{x}}}^{2} h+\frac{1}{4 !} D_{\widetilde{\mathbf{x}}}^{4} h+\cdots\right)
$$

Now we compute the value of $\widehat{\overline{\mathbf{y}}}$ by expanding each $\mathbf{y}^{(j)}$ in (24) in a Taylor series around $\overline{\mathbf{x}}$ as follows:

$$
\begin{aligned}
\widehat{\overline{\mathbf{y}}}= & \frac{1}{2\left(n_{x}+n_{\theta}\right)} \\
& \cdot \sum_{j=1}^{2\left(n_{x}+n_{\theta}\right)}\left(h(\overline{\mathbf{x}}, \bar{\theta})+D_{\widetilde{\mathbf{x}}} h+\frac{1}{2 !} D_{\widetilde{\mathbf{x}}}^{2(j)} h+\cdots\right) .
\end{aligned}
$$

Because $\widetilde{\widetilde{\mathbf{x}}}^{(j)}=-\widetilde{\widetilde{\mathbf{x}}}^{\left(n_{x}+n_{\theta}+j\right)}\left(j=1, \ldots, n_{x}+n_{\theta}\right)$, for any integer $k>0$, we have

$$
\begin{gathered}
\sum_{j=1}^{2\left(n_{x}+n_{\theta}\right)} D_{\widetilde{\mathbf{x}}^{(j)}}^{2 k+1} h=\sum_{j=1}^{2\left(n_{x}+n_{\theta}\right)}\left[\left(\left.\sum_{i=1}^{n_{x}+n_{\theta}} \widetilde{\mathbf{x}}_{i}^{(j)} \frac{\partial h}{\partial \check{\mathbf{x}}_{j}}\right|_{\check{\mathbf{x}}=\overline{\mathbf{x}}}\right)^{2 k+1}\right] \\
=\sum_{j=1}^{2\left(n_{x}+n_{\theta}\right)}\left[\left.\sum_{i=1}^{n_{x}+n_{\theta}}\left(\widetilde{\mathbf{x}}_{i}^{(j)}\right)^{2 k+1} \frac{\partial^{2 k+1} h}{\partial \check{\mathbf{x}}_{j}^{2 k+1}}\right|_{\check{\mathbf{x}}=\overline{\mathbf{x}}}\right] \\
=\sum_{i=1}^{n_{x}+n_{\theta}}\left[\left.\sum_{j=1}^{2\left(n_{x}+n_{\theta}\right)}\left(\widetilde{\tilde{\mathbf{x}}}_{i}^{(j)}\right)^{2 k+1} \frac{\partial^{2 k+1} h}{\partial \check{\mathbf{x}}_{j}^{2 k+1}}\right|_{\check{\mathbf{x}}=\overline{\mathbf{x}}}\right]=0 .
\end{gathered}
$$

Therefore, all of the odd terms in (32) evaluate to zero and we have

$$
\begin{aligned}
& \hat{\overline{\mathbf{y}}} \\
& =h(\overline{\mathbf{x}}, \bar{\theta}) \\
& \quad+\frac{1}{2\left(n_{x}+n_{\theta}\right)} \sum_{j=1}^{2\left(n_{x}+n_{\theta}\right)}\left(\frac{1}{2 !} D_{\widetilde{\mathbf{x}}}^{2} h+\frac{1}{4 !} D_{\widetilde{\mathbf{x}}}^{4} h+\cdots\right) .
\end{aligned}
$$

After some tedious calculation, we can verify that

$$
\begin{aligned}
& \frac{1}{2\left(n_{x}+n_{\theta}\right)} \sum_{j=1}^{2\left(n_{x}+n_{\theta}\right)} \frac{1}{2 !} D_{\widetilde{\mathbf{x}}}^{2} h=\left.\frac{1}{2} \sum_{i, j=1}^{n_{x}+n_{\theta}} \check{\mathbf{P}}_{i j} \frac{\partial^{2} h}{\partial \check{\mathbf{x}}_{i} \partial \check{\mathbf{x}}_{j}}\right|_{\check{\mathbf{x}}=\overline{\mathbf{x}}} \\
& =\frac{1}{2 !} E\left(D_{\widetilde{\mathbf{x}}}^{2} h\right) .
\end{aligned}
$$

It can be seen that $\widehat{\overline{\mathbf{y}}}$ (the approximated mean of y) matches the true mean of $\mathbf{y}$ correctly up to the third order.

Theorem 4. For random vector $\mathbf{x}$ with a symmetric $p d f$ around its mean, if the nonlinear function $\mathbf{y}=h(\mathbf{x}, \theta)$ contains unknown random parameter $\theta$, the approximated covariance of $\mathbf{y}$ via the improved unscented transformation (25) matches the true covariance of $\mathbf{y}$ correctly up to the third order.

Proof. The true covariance of $\mathbf{y}$ is given as

$$
\overline{\mathbf{P}}_{\mathbf{y}}=E\left[(\mathbf{y}-\overline{\mathbf{y}})(\mathbf{y}-\overline{\mathbf{y}})^{\prime}\right]
$$

By substituting (26) and (31) into (36) and using the same type of reasoning in the proof of Theorem 3 , we have that all of the odd-powered terms evaluate to zero. This leads to

$$
\begin{aligned}
\overline{\mathbf{P}}_{\mathbf{y}}= & E\left[D_{\widetilde{\mathbf{x}}} h\left(D_{\tilde{\mathbf{x}}} h\right)^{\prime}\right]+E\left[\frac{D_{\widetilde{\mathbf{x}}} h\left(D_{\widetilde{\mathbf{x}}}^{3} h\right)^{\prime}}{3 !}\right. \\
& \left.+\frac{D_{\widetilde{\mathbf{x}}}^{2} h\left(D_{\tilde{\mathbf{x}}}^{2} h\right)^{\prime}}{2 ! 2 !}+\frac{D_{\widetilde{\mathbf{x}}}^{3} h\left(D_{\tilde{\mathbf{x}}} h\right)^{\prime}}{3 !}\right]+E\left[\frac{D_{\widetilde{\mathbf{x}}}^{2} h}{2 !}\right] \\
& \cdot E\left[\frac{D_{\widetilde{\mathbf{x}}}^{2} h}{2 !}\right]^{\prime}+\cdots
\end{aligned}
$$

The first term on the right side of the equation above can be written as

$$
E\left[D_{\widetilde{\mathbf{x}}} h\left(D_{\widetilde{\mathbf{x}}} h\right)^{\prime}\right]=\mathbf{H} \check{\mathbf{P}} \mathbf{H}^{\prime}
$$

where $\mathbf{H}$ is the partial derivative matrix at $\check{\mathbf{x}}=\overline{\check{\mathbf{x}}}$. 
On the other hand, after some tedious calculation, the approximate covariance $\hat{\overline{\mathbf{P}}}_{\mathbf{y}}$ in (25) can be written as

$$
\begin{aligned}
\hat{\overline{\mathbf{P}}}_{\mathbf{y}} & =\frac{1}{2\left(n_{x}+n_{\theta}\right)} \sum_{j=1}^{2\left(n_{x}+n_{\theta}\right)}\left(D_{\widetilde{\mathbf{x}}(j)} h\right)\left(D_{\widetilde{\mathbf{x}}} h\right)^{\prime}+\text { HOT } \\
& =\frac{1}{2\left(n_{x}+n_{\theta}\right)} \\
& \cdot \sum_{j=1}^{2\left(n_{x}+n_{\theta}\right)} \sum_{i, k=1}^{n_{x}+n_{\theta}}\left(\widetilde{\mathbf{x}}_{i}^{(j)} \frac{\partial h(\overline{\tilde{\mathbf{x}}})}{\partial \check{\mathbf{x}}_{i}}\right)\left(\widetilde{\mathbf{x}}_{k}^{(j)} \frac{\partial h(\overline{\tilde{\mathbf{x}}})}{\partial \check{\mathbf{x}}_{k}}\right)^{\prime} \\
& + \text { HOT, }
\end{aligned}
$$

where HOT means higher-order terms (i.e., terms to the forth power and higher). Now recall that $\widetilde{\mathbf{x}}_{i}^{(j)}=-\widetilde{\mathbf{x}}_{i}^{\left(n_{x}+n_{\theta}+j\right)}$ and $\widetilde{\mathbf{x}}_{k}^{(j)}=-\widetilde{\mathbf{x}}_{k}^{\left(n_{x}+n_{\theta}+j\right)}$ for $j=1, \ldots, n_{x}+n_{\theta}$; therefore, the covariance approximation becomes

$$
\begin{aligned}
\hat{\overline{\mathbf{P}}}_{\mathbf{y}} & \\
= & \frac{1}{n_{x}+n_{\theta}} \sum_{j=1}^{n_{x}+n_{\theta}} \sum_{i, k=1}^{n_{x}+n_{\theta}}\left(\widetilde{\mathbf{x}}_{i}^{(j)} \frac{\partial h(\overline{\check{\mathbf{x}}})}{\partial \check{\mathbf{x}}_{i}}\right)\left(\widetilde{\widetilde{\mathbf{x}}}_{k}^{(j)} \frac{\partial h(\overline{\check{\mathbf{x}}})}{\partial \check{\mathbf{x}}_{k}}\right)^{\prime} \\
& +\mathrm{HOT}=\mathbf{H} \check{\mathbf{P}} \mathbf{H}^{\prime}+\text { HOT. }
\end{aligned}
$$

Comparing (40) with the true covariance of $\mathbf{y}$ from (37), we see that $\hat{\overline{\mathbf{P}}}_{\mathbf{y}}$ in (25) approximates the true covariance of $\mathbf{y}$ up to the third order.

3.2. Improved Unscented Kalman Filter. The improved unscented transformation developed in the previous subsection can be generalized to give the improved unscented Kalman filter for the discrete-time nonlinear system which contains random parameters, that is,

$$
\begin{aligned}
& \mathbf{x}_{k}=f\left(\mathbf{x}_{k-1}, \theta\right)+\mathbf{w}_{k}, \\
& \mathbf{y}_{k}=h\left(\mathbf{x}_{k}, \theta\right)+\mathbf{v}_{k},
\end{aligned}
$$

where the motion function $f(\cdot)$ and the measurement function $h(\cdot)$ are nonlinear with respect to not only the state vector $\mathbf{x}_{k}$ but also the random parameter $\theta$. The other notations are the same as that of model (1). Assume that the parameter $\theta \in$ $\mathbb{R}^{n_{\theta}}$ has known mean $\bar{\theta}$ and covariance $\mathbf{P}^{\theta}$ and is uncorrelated with the state vector $\mathbf{x}_{k}$ as well as the noise vectors $\mathbf{w}_{k}$ and $\mathbf{v}_{k}$.

Following the augment method of the improved unscented transformation, we give the augment model of the discrete-time nonlinear system (41) as follows:

$$
\begin{aligned}
& \check{\mathbf{x}}_{k}=f\left(\check{\mathbf{x}}_{k-1}\right)+\check{\mathbf{w}}_{k}, \\
& \mathbf{y}_{k}=h\left(\check{\mathbf{x}}_{k}\right)+\mathbf{v}_{k},
\end{aligned}
$$

where

$$
\begin{aligned}
\check{\mathbf{x}}_{k} & =\left[\mathbf{x}_{k}^{\prime}, \theta^{\prime}\right]^{\prime}, \\
\check{\mathbf{w}}_{k} & =\left[\check{\mathbf{w}}_{k}^{\prime}, \mathbf{0}^{\prime}\right]^{\prime} .
\end{aligned}
$$

With the initial condition (7), the improved unscented Kalman filter can be initialized as follows:

$$
\begin{aligned}
& \widehat{\check{\mathbf{x}}}_{0}^{+}=\left[\left(\widehat{\mathbf{x}}_{0}^{+}\right)^{\prime}, \bar{\theta}^{\prime}\right]^{\prime}, \\
& \check{\mathbf{P}}_{0}^{+}=\left(\begin{array}{cc}
\mathbf{P}_{0}^{+} & \mathbf{0} \\
\mathbf{0} & \mathbf{P}^{\theta}
\end{array}\right) .
\end{aligned}
$$

Suppose that, at time step $k, \widehat{\check{\mathbf{x}}}_{k-1}^{+}$and $\check{\mathbf{P}}_{k-1}^{+}$are given. For the sake of brevity, we denote $\check{n}=n_{x}+n_{\theta}$. The improved UKF algorithm can be summarized in Algorithm 5.

Algorithm 5 (the improved unscented Kalman filter).

(1) Choose $2 \check{n}$ sigma points $\widehat{\widetilde{\mathbf{x}}}_{k-1}^{(j)}$ as specified in (19) with appropriate changes, that is,

$$
\begin{aligned}
\check{\widetilde{\mathbf{x}}}_{k-1}^{(j)} & =\widehat{\widetilde{\mathbf{x}}}_{k-1}^{+}+\widetilde{\widetilde{\mathbf{x}}}_{k-1}^{(j)}, \quad j=1, \ldots, 2 \check{n}, \\
\widetilde{\widetilde{\mathbf{x}}}_{k-1}^{(j)} & =\left(\sqrt{\check{\check{n} \check{\mathbf{P}}_{k-1}^{+}}}\right)_{j}^{\prime}, \quad j=1, \ldots, \check{n}, \\
\widetilde{\mathbf{x}}_{k-1}^{(\check{n}+j)} & =-\widetilde{\mathbf{x}}_{k-1}^{(j)}, \quad j=1, \ldots, \check{n} .
\end{aligned}
$$

(2) Transform the sigma points $\widehat{\widetilde{\mathbf{x}}}_{k-1}^{(j)}$ into $\widehat{\widetilde{\mathbf{x}}}_{k}^{(j)}$ as follows:

$$
\widehat{\widetilde{\mathbf{x}}}_{k}^{(j)}=f\left(\widehat{\mathbf{x}}_{k-1}^{(j)}, \widehat{\theta}_{k-1}^{(j)}\right) \text {, }
$$

where $\widehat{\mathbf{x}}_{k-1}^{(j)}=\widehat{\widetilde{\mathbf{x}}}_{k-1}^{(j)}\left(1: n_{x}\right)$ and $\widehat{\theta}_{k-1}^{(j)}=\widehat{\mathbf{x}}_{k-1}^{(j)}\left(n_{x}+1: \check{n}\right)$.

(3) Combine the $\widehat{\hat{\mathbf{x}}}_{k}^{(j)}$ vectors to obtain the prediction estimate at time $k$

$$
\widehat{\widetilde{\mathbf{x}}}_{k}^{-}=\frac{1}{2 \check{n}} \sum_{j=1}^{2 \check{n}} \widetilde{\mathbf{x}}_{k}^{(j)}
$$

and the corresponding error covariance

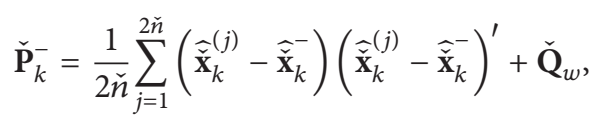

where $\check{\mathbf{Q}}_{w}=\left(\begin{array}{cc}\mathbf{Q}_{w} & 0 \\ 0 & 0\end{array}\right)$.

(4) Transform the sigma points $\widehat{\widetilde{\mathbf{x}}}_{k-1}^{(j)}$ into $\widehat{\tilde{\mathbf{y}}}_{k}^{(j)}$ vectors:

$$
\widehat{\tilde{\mathbf{y}}}_{k}^{(j)}=h\left(\widehat{\mathbf{x}}_{k-1}^{(j)}, \widehat{\theta}_{k-1}^{(j)}\right) .
$$

(5) Combine the $\widehat{\widehat{\mathbf{y}}}_{k}^{(j)}$ vectors to obtain the predicted measurement at time $k$

$$
\widehat{\tilde{\mathbf{y}}}_{k}=\frac{1}{2 \check{n}} \sum_{j=1}^{2 \check{\mathbf{y}}} \widetilde{\mathbf{y}}_{k}^{(j)}
$$


and the corresponding covariance

$$
\check{\mathbf{P}}_{k}^{y}=\frac{1}{2 \check{n}} \sum_{j=1}^{2 \check{n}}\left(\check{\check{\mathbf{y}}}_{k}^{(j)}-\widehat{\check{\mathbf{y}}}_{k}\right)\left(\check{\check{\mathbf{y}}}_{k}^{(j)}-\widehat{\check{\mathbf{y}}}_{k}\right)^{\prime}+\mathbf{Q}_{v} \text {. }
$$

(6) Estimate the cross covariance between $\widehat{\widetilde{\mathbf{x}}}_{k}^{-}$and $\widehat{\tilde{\mathbf{y}}}_{k}$ :

$$
\check{\mathbf{P}}_{k}^{x y}=\frac{1}{2 \check{n}} \sum_{j=1}^{2 \check{n}}\left(\hat{\check{\mathbf{x}}}_{k}^{(j)}-\widehat{\check{\mathbf{x}}}_{k}^{-}\right)\left(\hat{\check{\mathbf{y}}}_{k}^{(j)}-\widehat{\check{\mathbf{y}}}_{k}\right)^{\prime} .
$$

(7) The measurement update of the state estimate can be performed as follows:

$$
\begin{aligned}
& \check{\mathbf{K}}_{k}=\check{\mathbf{P}}_{k}^{x y}\left(\check{\mathbf{P}}_{k}^{y}\right)^{-1}, \\
& \check{\check{\mathbf{x}}}_{k}^{+}=\widehat{\check{\mathbf{x}}}_{k}^{-}+\check{\mathbf{K}}_{k}\left(\mathbf{y}_{k}-\check{\mathbf{y}}_{k}\right), \\
& \check{\mathbf{P}}_{k}^{+}=\check{\mathbf{P}}_{k}^{-}-\check{\mathbf{K}}_{k} \check{\mathbf{P}}_{k}^{y} \check{\mathbf{K}}_{k}^{\prime} .
\end{aligned}
$$

The proposed improved UKF method provides a suboptimal solution for the stochastic filtering problem of a nonlinear discrete-time dynamic system which contains random parameters. The main difference between the improved UKF algorithm and the normal UKF algorithm lies in the construction of the sigma points. The number of sigma points of the improved UKF algorithm is larger than that of the normal UKF algorithm, so generally the proposed improved UKF method requires higher computational complexity. However, the advantage of the improved UKF method is the increased accuracy of the state estimation which can be verified by the numerical results in the following section.

\section{An Application to Mobile Source Localization}

Consider a wireless sensor network with $n$ passive sensor nodes, distributed on a $2 \mathrm{D}$ plane, to localize one moving source. Let the state vector of the mobile source be $\mathbf{x}_{k}=$ $\left[\mathbf{u}_{k}^{\prime}, \mathbf{v}_{k}^{\prime}\right]^{\prime} \in \mathbb{R}^{4}$, which includes the position and velocity components.

Without loss of generality, let the first sensor be the reference. The TDOA measurement model [20] between sensor pair $i(i=2, \ldots, n)$ and 1 is

$$
t_{i 1}\left(\mathbf{u}_{k}\right)=\frac{\left\|\mathbf{u}_{k}-\mathbf{s}_{i}^{0}\right\|}{c}-\frac{\left\|\mathbf{u}_{k}-\mathbf{s}_{1}^{0}\right\|}{c}+\Delta t_{i k},
$$

where $\mathbf{s}_{i}^{0}$ is the true position of the $i$ th sensor, $r_{i}^{0}\left(\mathbf{u}_{k}\right)=$ $\left\|\mathbf{u}_{k}-\mathbf{s}_{i}^{0}\right\|$ is the true distance from the source to the $i$ th sensor, and $c$ is the signal propagation speed. The vector $\Delta \mathbf{t}_{k}=$ $\left[\Delta t_{2 k}, \ldots, \Delta t_{n k}\right]^{\prime}$ is zero-mean Gaussian noise with covariance $\mathbf{Q}_{t}$. In simplicity, (54) is expressed in the vector form

$$
\mathbf{t}_{k}=\left[t_{21}\left(\mathbf{u}_{k}\right), \ldots, t_{n 1}\left(\mathbf{u}_{k}\right)\right] .
$$

In practice, the true sensor positions $\mathbf{s}^{0}=\left[\mathbf{s}_{1}^{0 \prime}, \ldots, \mathbf{s}_{4}^{0 \prime}\right]^{\prime}$ are not known, and only their nominal values $\mathbf{s}=$
TABLE 1: Nominal positions (in meters) of sensors.

\begin{tabular}{lcc}
\hline Sensor number $i$ & $x_{i}$ & $y_{i}$ \\
\hline 1 & 100 & 100 \\
2 & 100 & -100 \\
3 & -100 & -100 \\
4 & -100 & 100 \\
\hline
\end{tabular}

$\left[\mathbf{s}_{1}^{\prime}, \mathbf{s}_{2}^{\prime}, \ldots, \mathbf{s}_{4}^{\prime}\right]^{\prime}$ are available for source localization. More precisely, $\mathbf{s}_{i}^{0}$ and $\boldsymbol{s}_{i}$ are related by

$$
\mathbf{s}_{i}^{0}=\mathbf{s}_{i}+\Delta \mathbf{s}_{i}
$$

where $\Delta \mathbf{s}_{i}$ is the sensor position noise and the corresponding noise vector $\Delta \mathbf{s}=\left[\Delta \mathbf{s}_{1}^{\prime}, \Delta \mathbf{s}_{2}^{\prime}, \ldots, \Delta \mathbf{s}_{n}^{\prime}\right]^{\prime}$ is assumed to be zeromean Gaussian with covariance matrix $\mathbf{Q}_{s}$.

Because the TDOA measurement vector (55) is nonlinear with respect to the state vector $\mathbf{x}_{k}$ as well as the true sensor locations $\mathbf{s}^{0}$, the mobile source localization problem can be described as the following discrete-time nonlinear system:

$$
\mathbf{t}_{k}=\mathbf{f}\left(\mathbf{x}_{k}, \mathbf{s}^{0}\right)+\Delta \mathbf{t}_{k},
$$

where $\mathbf{s}^{0}$ can be regarded as unknown random parameters. Thus, we can apply the proposed improved UKF method to dynamically estimate the state vector.

In order to show the performance of the proposed improved UKF method, we apply it to two localization scenarios and compare it with the normal UKF method, which ignores the random uncertainties in the sensor locations, through Monte Carlo simulations.

4.1. Scenario 1. This simulation scenario contains $n=4$ sensors, and their nominal positions are given in Table 1 . The motion model of the mobile source is assumed to be a nearly constant velocity model as

$$
\begin{aligned}
\mathbf{x}_{k}= & \left(\begin{array}{cccc}
1 & 0 & 0.01 & 0 \\
0 & 1 & 0 & 0.01 \\
0 & 0 & 1 & 0 \\
0 & 0 & 0 & 1
\end{array}\right) \mathbf{x}_{k-1} \\
& +\left(\begin{array}{cc}
0.01 & 0 \\
0 & 0.01 \\
0.1 & 0 \\
0 & 0.1
\end{array}\right) \mathbf{w}_{k-1}
\end{aligned}
$$

where $\mathbf{w}_{k-1}$ is zero-mean Gaussian noise with covariance matrix $\mathbf{Q}_{w}=0.01 \mathbf{I}$. The initial value of the state $\mathbf{x}_{0}$ is a Gaussian vector with zero mean and covariance $\mathbf{P}_{0}^{+}=$ $\operatorname{diag}\{100,100,0.01,0.01\}$. The trajectory of the mobile source in 100 time instants is shown in Figure 1.

The TDOA measurement covariance $\mathbf{Q}_{t}=(0.5 / c)^{2} \mathbf{T}$, where $\mathbf{T}$ is the $(n-1) \times(n-1)$ matrix with 1 in the diagonal elements and 0.5 otherwise. The localization accuracy is evaluated by the root of mean squared error which is defined 


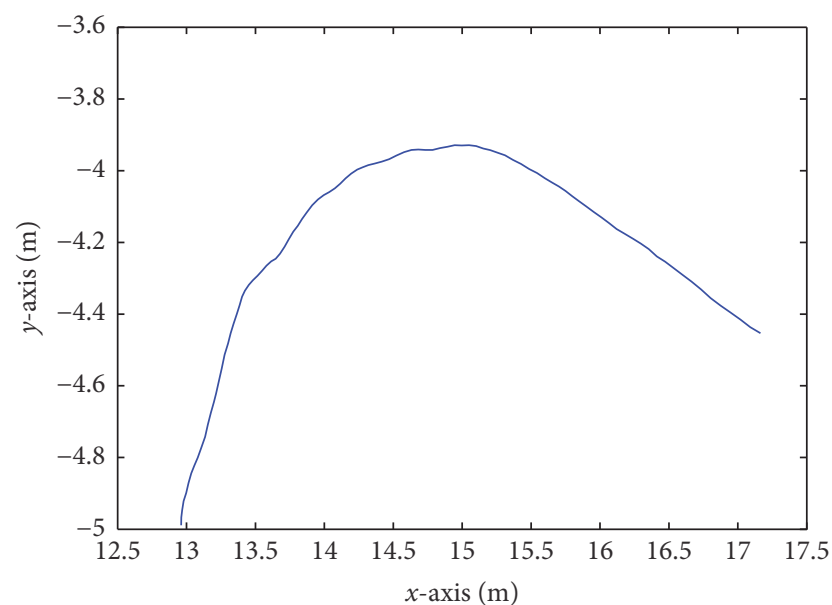

FIgURE 1: The trajectory of the mobile source in Scenario 1.

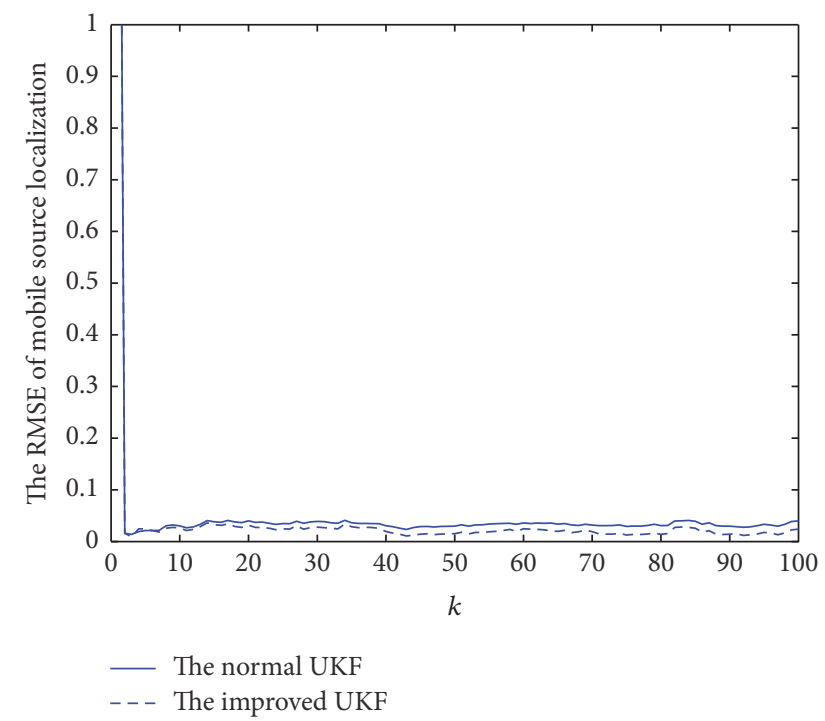

Figure 2: Comparison of the mobile localization accuracy with low level sensor position uncertainties in Scenario 1.

as $\operatorname{RMSE}\left(\mathbf{u}_{k}\right)=\sqrt{\sum_{l=1}^{L}\left\|\widehat{\mathbf{u}}_{k}-\mathbf{u}_{k}\right\|^{2} / L}$, where $\widehat{\mathbf{u}}_{k}=\widehat{\mathbf{x}}_{k}(1: 2)$ is the estimate of the source position at ensemble $l$ and $L=1000$ is the number of ensemble runs.

In the Monte Carlo simulations, we consider three sensor position uncertainty levels which are referred to as the low level with $\sigma_{s}=0.1$, the moderate level with $\sigma_{s}=1$, and the high level with $\sigma_{s}=2$.

Figure 2 plots the simulation results with time instant varying from 1 to 100 when the sensor position uncertainty level is low. It is evident from Figure 2 that RMSE of the proposed improved UKF method (represented by dashed line) is smaller than that of the normal UKF approach (represented by solid line) which ignores the sensor position uncertainties.

Figure 3 plots the RMSEs of the two methods in the case of moderate sensor position uncertainty level, that is, $\sigma_{s}=$ 1. We can see that both of them are generally larger than

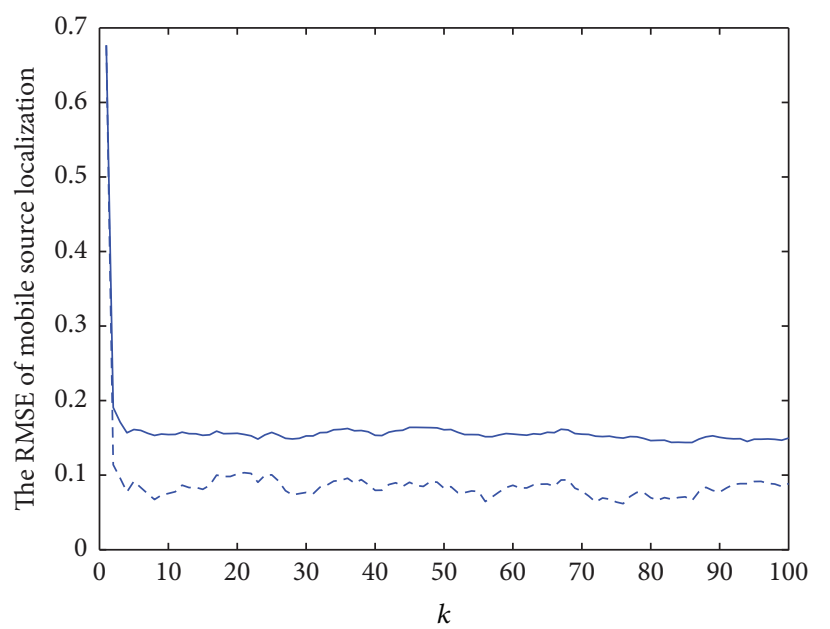

- The normal UKF

- - The improved UKF

FIgURE 3: Comparison of the mobile localization accuracy with mild level sensor position uncertainties in Scenario 1.

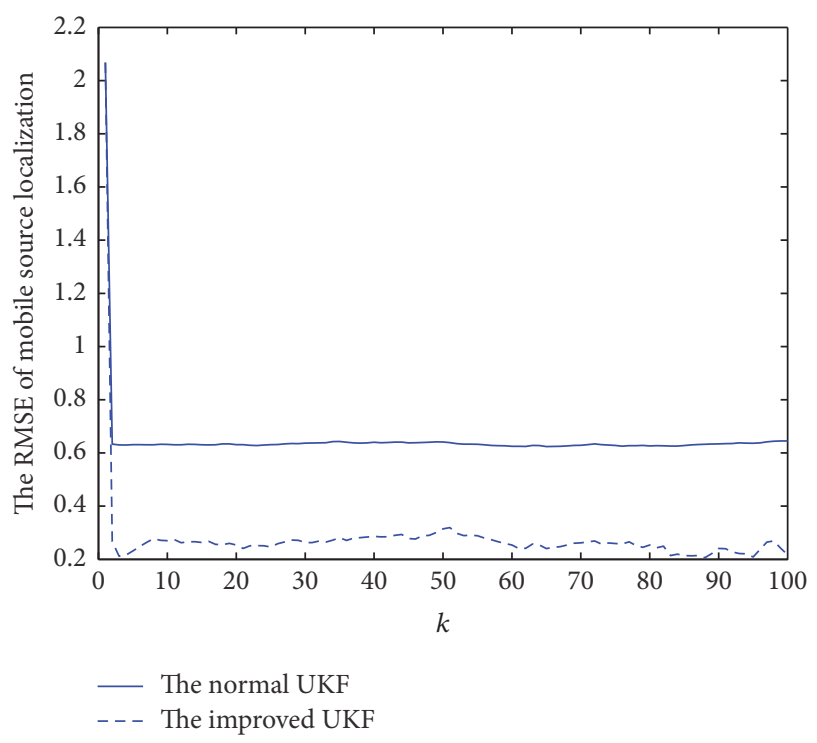

FIGURE 4: Comparison of the mobile localization accuracy with high level sensor position uncertainties in Scenario 1.

the corresponding RMSEs in Figure 2. As we expected, the performance of the proposed improved UKF method is much better than that of the normal UKF approach. In addition, the improvement is larger than that of the low level case in Figure 2.

Finally, we take the high sensor position uncertainty level, that is, $\sigma_{s}=2$. The numerical simulation results are illustrated in Figure 4, which indicates that the RMSE of the normal $\mathrm{UKF}$ approach is nearly twice as much as that of the proposed improved UKF method. Comparing the results in Figures 24 , we can see that the influence of the random sensor position uncertainties on the localization performance is quite evident in Scenario 1. The proposed improved UKF method can 


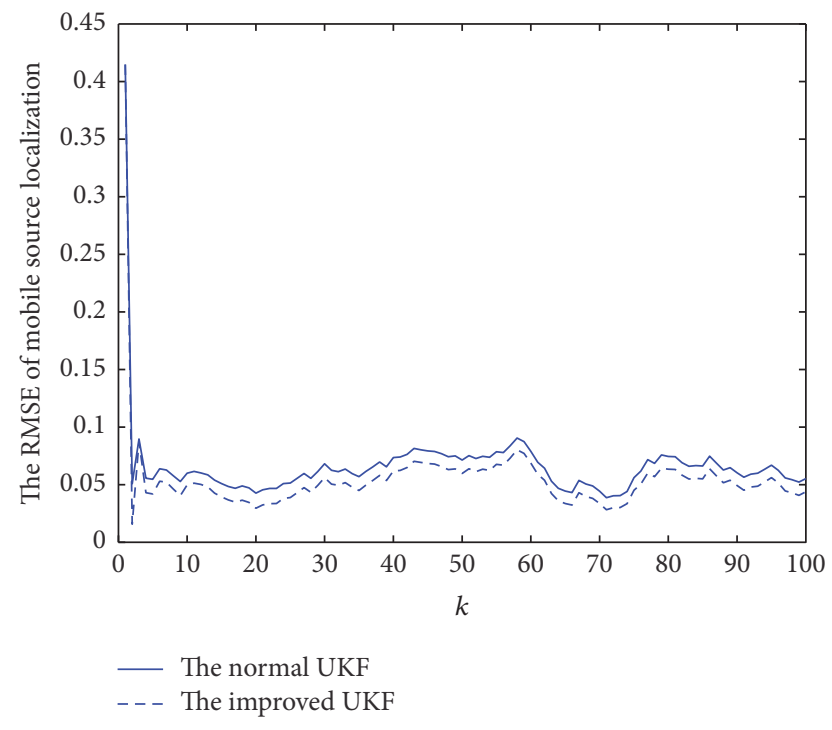

FIGURE 5: Comparison of the mobile localization accuracy with low level sensor position uncertainties in Scenario 2.

efficiently decrease the RMSE, especially when the sensor position uncertainty level is high.

4.2. Scenario 2. In this simulation scenario, we consider the sensor geometry is random and the number of sensors $n=4$. The true position of each sensor is uniformly distributed in a square of $[-100,100] \mathrm{m} \times[-100,100] \mathrm{m}$ at each ensemble in Monte Carlo simulations. The TDOA measurement covariance $\mathbf{Q}_{t}=(0.1 / c)^{2} \mathbf{T}$. The motion model and the initial value of the state are generally the same as those of Scenario 1.

In the first case, the sensor position uncertainty level is considerably small, that is, $\sigma_{s}=0.1$. Figure 5 plots the RMSE of the normal UKF approach and that of the proposed improved UKF method with time instant varying from 1 to 100 , which are calculated from $L=1000$ Monte Carlo runs. It can be seen that the RMSE of the proposed improved UKF method is smaller than that of the normal UKF approach.

Then, we consider a moderate sensor position uncertainty level, that is, $\sigma_{s}=1$. Figure 6 plots the RMSEs of the two methods in this case, where we can see that the performance of the proposed improved UKF method is still better than that of the normal UKF approach with a larger improvement as in Figure 5.

For the high sensor position uncertainty level, that is, $\sigma_{s}=2$, the simulation results are illustrated in Figure 7, which shows that the RMSE of the normal UKF approach is considerably larger than that of the proposed improved UKF method.

The Monte Carlo simulation results in Figures 5-7 reveal that the RMSEs of the proposed improved UKF are consistently smaller than those of the normal UKF approach, which corroborates that the proposed improved UKF method can generally improve the estimation accuracy of the nonlinear dynamic system when it contains random parameters.

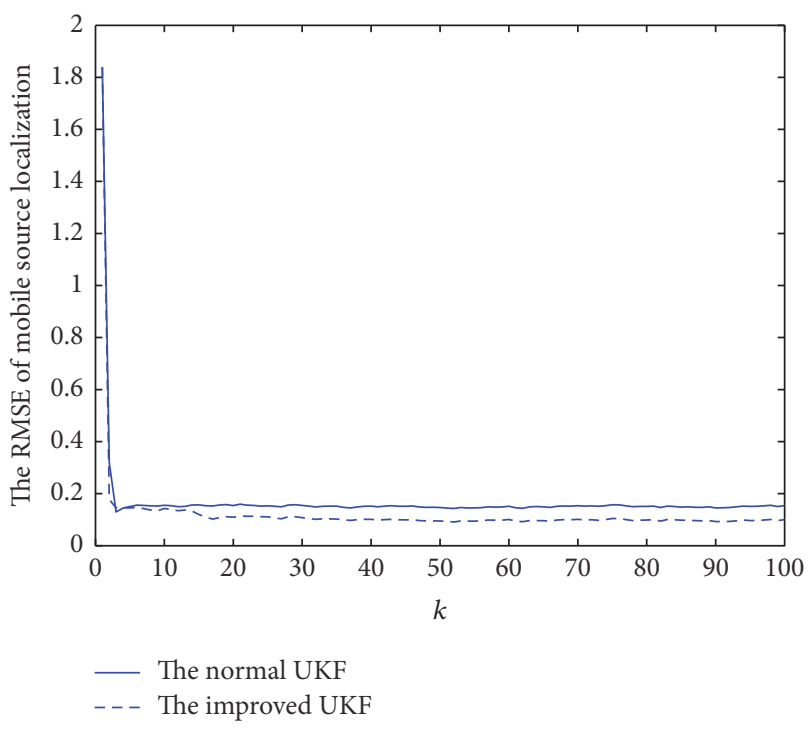

FIGURE 6: Comparison of the mobile localization accuracy with mild level sensor position uncertainties in Scenario 2.

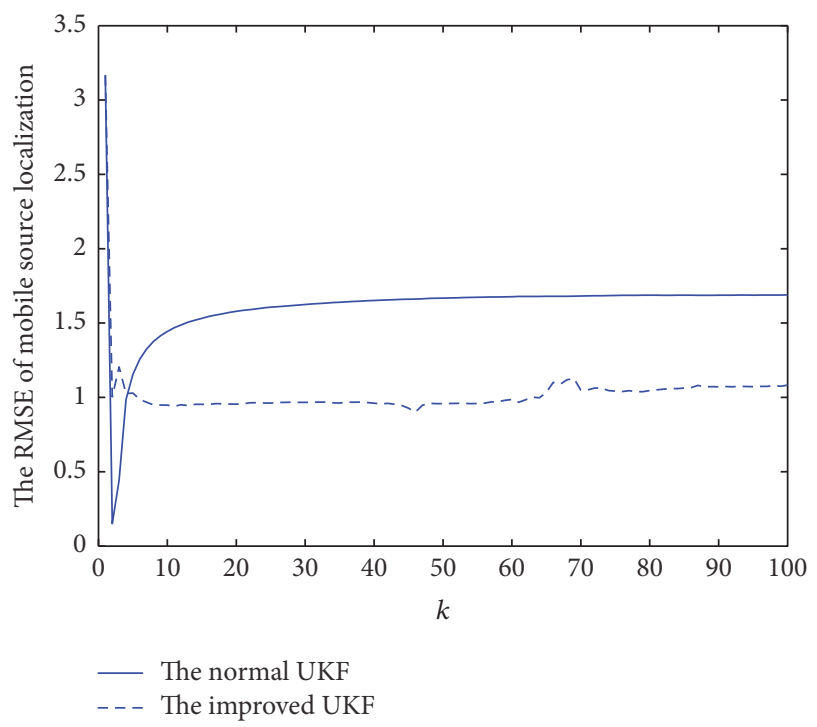

FIGURE 7: Comparison of the mobile localization accuracy with high level sensor position uncertainties in Scenario 2.

\section{Conclusions}

In this paper, an improved unscented Kalman filtering method is explored for discrete nonlinear dynamic systems with random parameters. By augmenting the random parameters into the state vector, we enlarge the number of sigma points in the improved unscented transformation. Theoretical analysis indicates that the approximated mean and covariance via the improved unscented transformation match the true values correctly up to the third order of Taylor series expansion. An application to the mobile source localization using TDOA measurements is provided, where the sensor positions suffer from random uncertainties. The Monte Carlo 
simulation results show that the proposed improved UKF method can considerably improve the estimation accuracy.

\section{Competing Interests}

The authors declare that there are no competing interests regarding the publication of this paper.

\section{Acknowledgments}

This work was supported by the Fundamental Research Funds for the Central Universities, Southwestern University of Finance and Economics, under Grant no. JBK1507059, and Southwest University for Nationalities, under Grant no. 2016 NGJPY05.

\section{References}

[1] R. E. Kalman, "A new approach to linear filtering and prediction problems," Journal of Basic Engineering, vol. 82, no. 1, pp. 35-45, 1960.

[2] G. Bolzon, R. Fedele, and G. Maier, "Parameter identification of a cohesive crack model by Kalman filter," Computer Methods in Applied Mechanics and Engineering, vol. 191, no. 25-26, pp. 2847-2871, 2002.

[3] M. Ball and A. Wood, "Trend growth in post-1850 British economic history: the Kalman filter and historical judgment," Journal of the Royal Statistical Society, vol. 45, no. 2, Article ID 143152, pp. 143-152, 1996.

[4] W. L. De Koning, "Optimal estimation of linear discrete-time systems with stochastic parameters," Automatica, vol. 20, pp. 113-115, 1984.

[5] B. Sinopoli, L. Schenato, M. Franceschetti, K. Poolla, M. I. Jordan, and S. S. Sastry, "Kalman filtering with intermittent observations," IEEE Transactions on Automatic Control, vol. 49, no. 9, pp. 1453-1464, 2004.

[6] Y. Luo, Y. Zhu, D. Luo, J. Zhou, E. Song, and D. Wang, “Globally optimal multisensor distributed random parameter matrices kalman filtering fusion with applications," Sensors, vol. 8, no. 12, pp. 8086-8103, 2008.

[7] S. Chen, Y. Li, G. Qi, and A. Sheng, "Adaptive Kalman estimation in target tracking mixed with random one-step delays, stochastic-bias measurements, and missing measurements," Discrete Dynamics in Nature and Society, vol. 2013, Article ID 716915, 14 pages, 2013.

[8] S. Chen, Z. Zhou, and S. Li, "An efficient estimate and forecast of the implied volatility surface: a nonlinear Kalman filter approach," Economic Modelling, vol. 58, pp. 655-664, 2016.

[9] S. Bolognani, L. Tubiana, and M. Zigliotto, "Extended Kalman filter tuning in sensorless PMSM drives," IEEE Transactions on Industry Applications, vol. 39, no. 6, pp. 1741-1747, 2003.

[10] J. J. LaViola Jr., "A comparison of unscented and extended kalman filtering for estimating quaternion motion," in Proceedings of the American Control Conference, pp. 2435-2440, Denver, Colo, USA, June 2003.

[11] X. Qu and L. Xie, "Recursive source localisation by time difference of arrival sensor networks with sensor position uncertainty," IET Control Theory and Applications, vol. 8, no. 18, pp. 2305-2315, 2014.
[12] S. J. Julier, J. K. Uhlmann, and H. F. Durrant-Whyte, "New approach for filtering nonlinear systems," in Proceedings of the American Control Conference, pp. 1628-1632, June 1995.

[13] S. J. Julier and J. K. Uhlmann, "Unscented filtering and nonlinear estimation," Proceedings of the IEEE, vol. 92, no. 3, pp. 401422, 2004.

[14] Q. Pan, F. Yang, and L. Ye, "Survey of a kind of non linear filters UKF," Control and Decision, vol. 20, no. 5, pp. 481-489, 2005.

[15] C. Eberle and C. Ament, "The Unscented Kalman Filter estimates the plasma insulin from glucose measurement," BioSystems, vol. 103, no. 1, pp. 67-72, 2011.

[16] S. J. Julier, "Some relations between extended and unscented Kalman filters," IEEE Transactions on Signal Processing, vol. 60, no. 2, pp. 545-555, 2012.

[17] H. M. T. Menegaz, J. Y. Ishihara, G. A. Borges, and A. N. Vargas, "A systematization of the unscented Kalman filter theory," IEEE Transactions on Automatic Control, vol. 60, no. 10, pp. 25832598, 2015.

[18] D. Simon, Optimal State Estimation: Kalman, H Infinity, and Nonlinear Approaches, Wiley-Interscience, 2006.

[19] K. W. Lui, F. K. Chan, and H. C. So, "Semidefinite programming approach for range-difference based source localization," IEEE Transactions on Signal Processing, vol. 57, no. 4, pp. 1630-1633, 2009.

[20] X. Qu and L. Xie, "An efficient convex constrained weighted least squares source localization algorithm based on TDOA measurements," Signal Processing, vol. 119, pp. 142-152, 2016.

[21] G. Yanni and W. Qi, "The nonsequential fusion method for localization from unscented kalman filter by multistation array buoys," Discrete Dynamics in Nature and Society, vol. 2016, Article ID 7670609, 8 pages, 2016.

[22] A. H. Jazwinski, Stochastic Processes and Filtering Theory, Academic Press, Cambridge, Mass, USA, 1970.

[23] F. Daum, "Nonlinear filters: beyond the kalman filter," IEEE Aerospace and Electronic Systems Magazine, vol. 20, no. 8, pp. 57-69, 2005. 


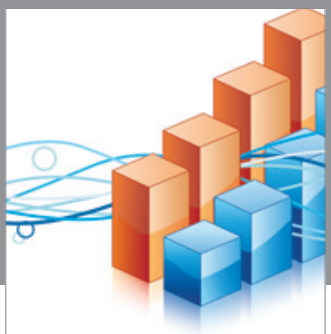

Advances in

Operations Research

vatem alat4

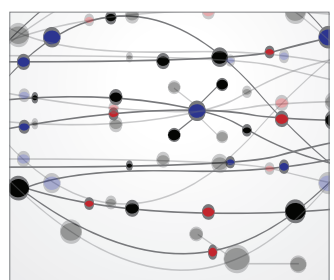

\section{The Scientific} World Journal
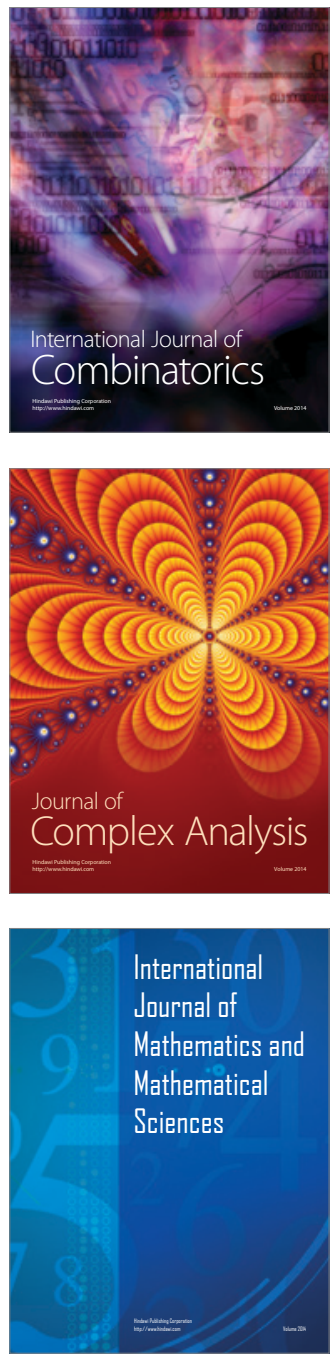
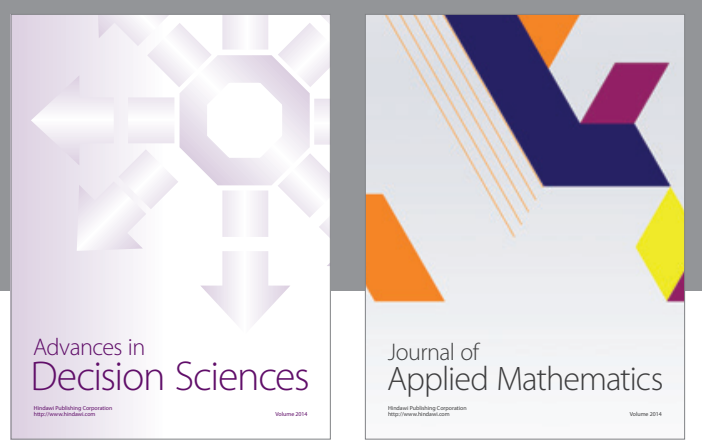

Algebra

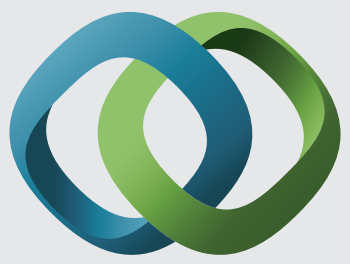

\section{Hindawi}

Submit your manuscripts at

https://www.hindawi.com
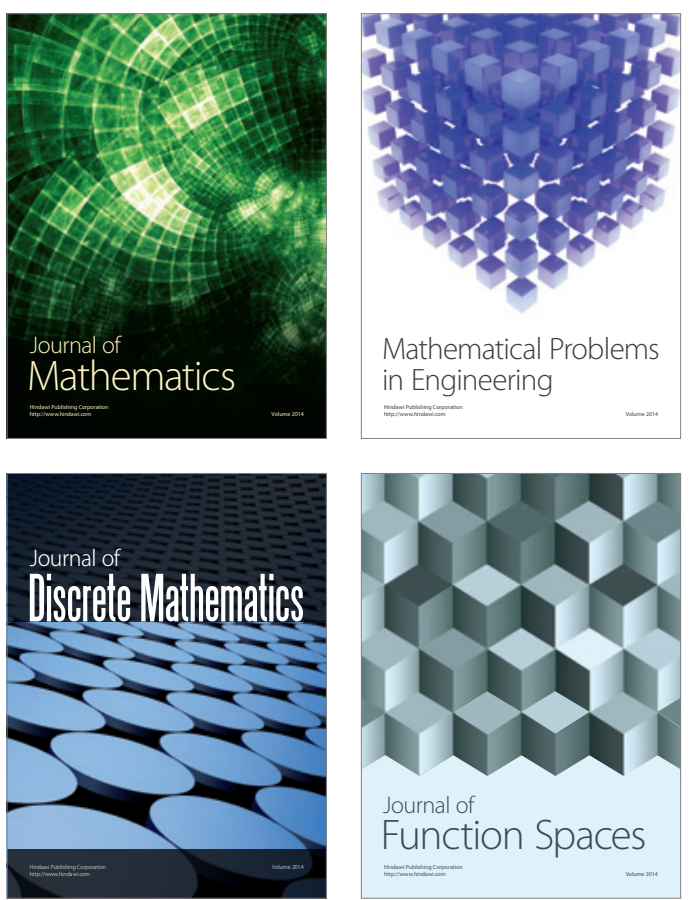

Mathematical Problems in Engineering
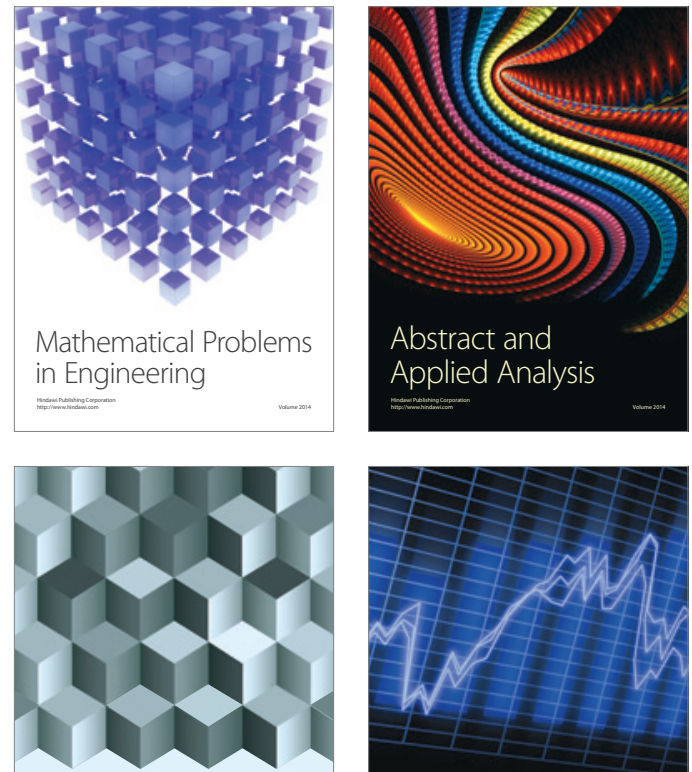

Journal of

Function Spaces

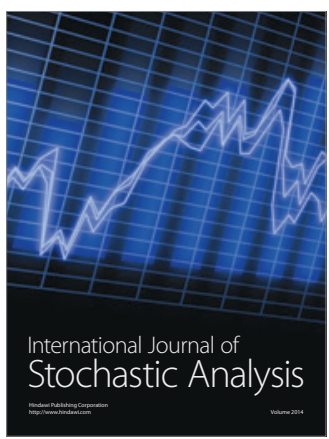

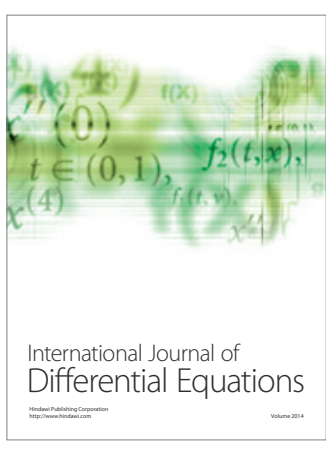
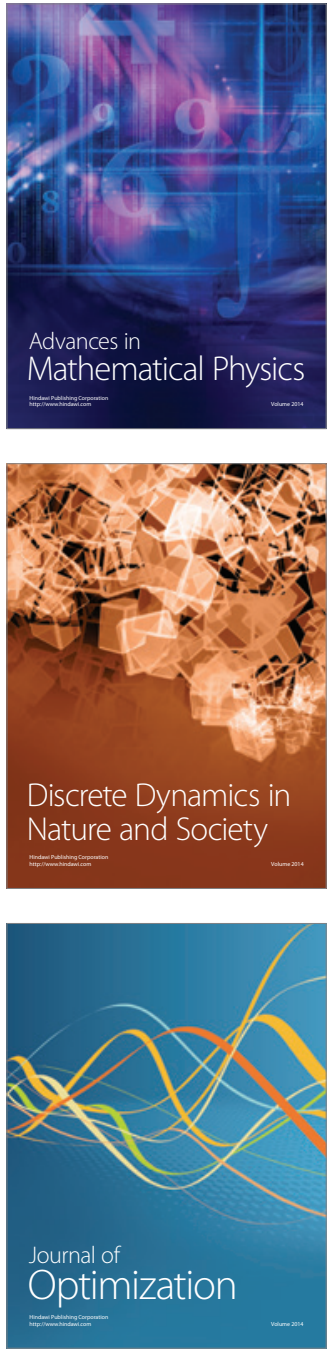